\title{
Mortalidade por doença de Chagas no Estado de São Paulo (Brasil): subsídios para o planejamento da assistência ao chagásico
}

\author{
Mortality due to Chagas disease in the State of S. Paulo, Brazil: parameter for \\ the establishment of adequate health care for the chagasic patient
}

\author{
Julio Litvoc*, Dalva Marli Valério Wanderley**, Luiz Marcelo Aranha Camargo***
}

\begin{abstract}
LITVOC, J. et al. Mortalidade por doença de Chagas no Estado de São Paulo (Brasil): subsídios para o planejamento da assistência ao chagásico. Rev. Saúde públ., S. Paulo, 26: 59-65, 1992. Foram analisadas as informações existentes $\mathrm{em} 1.646$ declarações de óbito de residentes no Estado de São Paulo, falecidos em 1987, identificando-se a causa básica segundo as regras de seleção utilizadas internacionalmente. Foram calculados o indicador "Anos Potenciais de Vida Perdidos" e a mortalidade proporcional para a doença de Chagas e para a doença isquêmica do coração, considerando somente a população de 15 a 70 anos. A doença de Chagas csteve presente como causa básica em $0,9 \%$ da totalidade dos óbitos do Estado, mantendo tendência já identificada desde 1977. A participação proporcional da doença de Chagas no conjunto dos anos potenciais de vida perdidos do Estado, foi $1,1 \%$, e da doença isquêmica do coração foi de $2,4 \%$, enquanto que em termos de mortalidade proporcional os valores foram $1,2 \%$ c $4,0 \%$, respectivamente. A distribuição das pessoas segundo região de residência mostra que o maior número de óbilos ocorreu na Grande São Paulo, enquanto que Ribeirão Preto apresentou o coeficiente mais elevado; $20,8 \%$ dos óbitos do Estado ocorreram em municípios diferentes daquele em que a pessoa residia, tendo essa taxa de evasão variado de $17,8 \%$ a $29,1 \%$ nas diversas regiōes. A comparação desses dois subgrupos mostrou uma maior diferença em relação à variável sexo, una vez que $22,8 \%$ dos homens faleceram num município diferente, tendo sido verificado esse fato em $17,4 \%$ das mulheres. Confirmou-se a importância desse quadro no Estado de São Paulo, e ao mesmo tempo identificou-se informações relativas à distribuição da doença $\mathrm{em}$ várias regiōes, quer $\mathrm{em}$ termos de se conhecer $\circ$ risco de mortalidade da população adulta, quer em termos de planejamento de alenção ao chagásico.
\end{abstract}

Descritores: Tripanossomose sul-americana, mortalidade. Distribuição espacial. Assistência médica.

\section{Introduçáo}

Com a tendência, no Brasil, de redução do número de casos novos de transmissão vetorial da doença de Chagas - tendência essa que se concretizou de forma mais intensa no Estado de São Paulo a partir da década de 70 - manifesta-se de forma mais premente a necessidade de sc aperfeiçoar o atendimento clínico e cirúrgico ao contingente de pessoas infectadas ou docntes, quer $\mathrm{cm}$ nivel de

\footnotetext{
* Deparamento de Medicina Preventiva da Faculdade de Medicina da Universidade de São Paulo, SP - 13rasil

** Superintendência de Controle de Endemias (SUCEN) da Secretaria do Estado da Saúde - São Paulo, SP - Brasil.

*** Departamento de Parasitologia do Instituto de Ciências Biomédicas da Universidade de São Paulo - São Paulo, SP - Brasil.
}

Separatas/Reprints: J. Litvoc - Av. Dr. Amaldo, 455 - 01246 903 - São Paulo, SP - Brasil.

Publicação financiada pela FAPESP. Processo Saúde Colctiva 91/4994-0. assistência primária, quer em nível de um atendimento mais especializado $3,4,5$.

Nesse sentido, considerou-se como uma das etapas do planejamento de assistência ao chagásico o conhecimento da freqüência de indivíduos chagásicos na população, fato quc permite estabelecer um dimensionamento do problema e fornecer elementos para uma futura avaliação.

O conhecimento sobre a frequiência dos indivíduos chagásicos tcm sido adquirido, no Estado de São Paulo, pclos estudos de morbidade e de mortalidade, estudos complementares que focalizem momentos e aspectos diferenciados da doença de Chagas. Comparando-se os procedimentos efetuados nos dois tipos de estudos, observa-se que as pesquisas de morbidade apresentam maior precisão na definição do indivíduo chagásico, mas, em contrapartida, apesar de se ter examinado grupos significativos - como é o caso dos grupos profissionais, de doadores de sangue e hospitalizados -, não foram cfeluadas em amostras representativas de árcas de São Paulo ${ }^{1,5,6,12}$. 
Estes estudos - com a totalidade da população ou com amostras representativas - foram realizados em pesquisas de mortalidade, restringindo-se, no entanto, em termos espaciais, ao Estado de São Paulo como um todo ou a algumas áreas municipais, como as cidades de São Paulo, Ribeirão Preto e Botucatu ${ }^{7,10}$. Dentre os estudos sobre mortalidade, destacamos o trabalho do Ministério da Saúde ${ }^{9}$ que publica desde 1977 informações sobre os óbitos ocorridos no Brasil, permitindo, ao leitor, elaborar uma tendência histórica das doenças relatadas nesta publicação. Assim sendo, observa-se que de 1977 a 1986 ocorreram no Estado de São Paulo, em média, 1.537 óbitos anuais nos quais a doença foi identificada como causa básica, sendo que a participação da tripanossomíase americana na mortalidade proporcional foi da ordem de $0,9 \%$; os valores específicos para cada ano variaram de 1.455 a 1.615 . Outra característica desses estudos é a utilização restrita que fazem das informações constantes da declaração de óbito, restringindo-se ao aproveitamento das importantes variáveis sexo e idade.

Assim, no presente trabalho foi analisada a totalidade das declarações de óbito do Estado de São Paulo, no ano de 1987, nas quais houve menção à presença da doença de Chagas, com os seguintes propósitos: caracterizar a magnitude do problema no Estado de São Paulo; conhecer a distribuição espacial dos óbitos, segundo as várias regiōes do Es- tado; utilizar algumas variáveis constantes da declaração de óbito, tradicionalmente não utilizadas nos estudos de mortalidade sobre doença de Chagas.

\section{Material e Método}

As análises provêm das informações existentes em 1.646 declaraçð̃es de óbito de pessoas residentes no Estado de Såo Paulo, falecidas em 1987, nas quais houve referência à doença de Chagas em qualquer item do atestado de óbito (como causa básica de morte ou associada). Para a identificação da causa básica aplicaram-se as regras para seleção utilizadas internacionalmente ${ }^{2}$. $O$ material foi obtido a partir de levantamento manual em arquivos da Fundação Sistema Estadual de Análise de Dados - SEADE.

As variávcis codificadas foram as seguintes: sexo, idade, município de residência, local de ocorrência do óbito e forma clínica da doença de Chagas. Apenas a variável sexo apresenta as categorias já delineadas na própria declaração de óbito; as formas clínicas identificadas correspondem as categorias de 4 dígitos da Classificação Internacional de Doenças, Revisão $1975^{2}$. Para se analisar a distribuição da doença consideramos a divisão do Estado utilizada pela Superintendência de Controle de Endemias (SUCEN), da Secretaria de Estado da Saúde de São Paulo, em trabalho de campo, inclusive no controle da doença de Chagas (Figura).

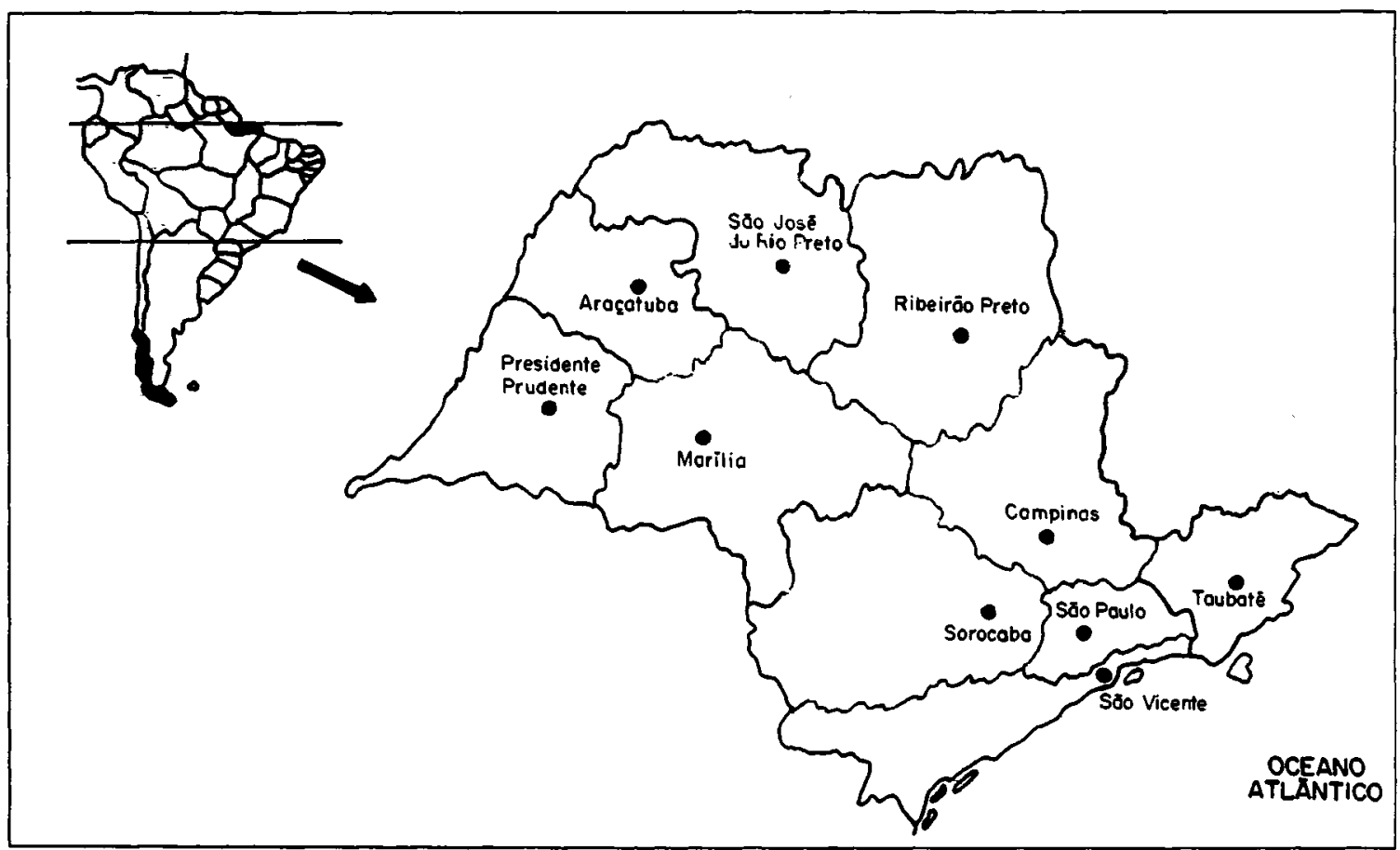

Figura: Divisão Regional da Superintêndencia de Controle de Endemias (SUCEN), São Paulo, Brasil 
Para o cálculo dos Anos Potenciais de Vida Perdidos (APVP) foi usado o método apresentado por Romeder e McWhinnie ${ }^{11}$ segundo Laurenti ${ }^{8}$ e que consiste na totalização do número de mortes, em cada idade (entre 1 e 70 anos), multiplicado pelos anos remanescentes de vida até a idade de 70 anos.

As informaçōes foram transcritas para um micro-computador de 16 bytes da Prológica, Solution 16, com Winchester de 20 megabytes, utilizandose para tal o aplicativo D-Base-III.

\section{Resultados}

Foram identificadas 1.646 declarações onde estava mencionado o evento doença de Chagas, tendo esta sido considerada como causa básica, em 1.516 declarações e em 130, como contributória. Nos resultados que serão apresentados a seguir, consideraram-se apenas as declaraçōes onde a doença de Chagas foi relatada como causa básica. O coeficiente para o Estado foi de 4,9 por 100.000 habitantes e a mortalidade proporcional de $0,9 \%$.

$\mathrm{Na}$ Tabela 1, pode-se observar que o número de homens falecidos, tendo como causa básica a doença de Chagas, é supcrior ao de mulheres, e que a proporção de óbitos é, em geral, crescente com a idade.

Na Tabela 2, estão descritos os APVP para o conjunto que faleceu por doença de Chagas $(23.757,5)$ e para o conjunto que faleceu por doença isquêmica do coração (DIC) (50.480), cálculos efetuados considerando-se a faixa etária de 15 a 70 anos. A participação proporcional da docnça de Chagas no conjunto dos APVP do Estado é de
$1,1 \%$, e a participação da DIC é de $2,4 \%$, enquanto que em termos de mortalidade proporcional, considcrando-se também a faixa etária de 15 a 70 anos, os valores são de 1,2 e $4,0 \%$, respectivamente.

A distribuição dos óbitos segundo local de residência, nas diferentes regiōes do Estado, é apresentada na Tabela 3 , onde se vê que a região que concentra maior número de pessoas ć a Grande São Paulo ( $36,3 \%$ dos óbitos), enquanto que Ribeirão Preto apresenta coeficiente mais elcvado, embora bastante próximo de São José do Rio Preto. Em 16

Tabela 1. Distribuição dos indivíduos falecidos com doença de Chagas, segundo sexo e faixa etária. Estado de São Paulo, 1987

\begin{tabular}{ccccc}
\hline & \multicolumn{3}{c}{ Sexo } & \multicolumn{2}{c}{ Total } \\
Faixa Etária & Masculino & Feminino & $N^{0}$ & $\%$ \\
\hline$<1$ & - & - & - & - \\
$1-4$ & - & - & - & - \\
$5-9$ & - & - & - & - \\
$10-14$ & - & - & - & - \\
$15-19$ & 8 & 4 & 12 & 0,8 \\
$20-24$ & 9 & 5 & 14 & 0,9 \\
$25-29$ & 29 & 9 & 38 & 2,5 \\
$30-34$ & 56 & 25 & 81 & 5,3 \\
$35-39$ & 85 & 21 & 106 & 7,0 \\
$40-44$ & 82 & 39 & 121 & 8,0 \\
$45-49$ & 97 & 65 & 162 & 10,7 \\
$50-54$ & 116 & 72 & 188 & 12,4 \\
$55-59$ & 127 & 66 & 193 & 12,7 \\
$60-64$ & 106 & 70 & 176 & 11,6 \\
$65-69$ & 91 & 57 & 148 & 9,8 \\
70 e + & 156 & 105 & 261 & 17,2 \\
Ignorado & 10 & 6 & 16 & 1,1 \\
\hline Total & 972 & 544 & 1.516 & 100,0 \\
& $(64,1 \%)$ & $(35,9 \%)$ & & \\
\hline
\end{tabular}

Tabela 2. Anos potenciais de vida perdidos (APVP) entre 15 e 70 anos, segundo todas as causas, doença de Chagas e doença isquêmica do coração. Estado de São Paulo, 1987

\begin{tabular}{|c|c|c|c|c|c|c|c|}
\hline \multirow[b]{2}{*}{ Grupo Etário } & \multirow[b]{2}{*}{$\begin{array}{c}\text { Anos } \\
\text { remanescentes }\end{array}$} & \multicolumn{2}{|c|}{ Todas as causas } & \multicolumn{2}{|c|}{ Doença de Chagas } & \multicolumn{2}{|c|}{ Doença isquêmica do coração } \\
\hline & & $N^{8}$ de óbitos & APVP & $N^{2}$ de óbitos & APVP & $N^{2}$ de óbitos & APVP \\
\hline $\begin{array}{l}15-19 \\
20-24 \\
25-29 \\
30-34 \\
35-39 \\
40-44 \\
45-49 \\
50-54 \\
55-59 \\
60-64 \\
65-69\end{array}$ & $\begin{array}{l}52,5 \\
47,5 \\
42,5 \\
37,5 \\
32,5 \\
27,5 \\
22,5 \\
17,5 \\
12,5 \\
7,5 \\
2,5\end{array}$ & $\begin{array}{r}4.015 \\
5.589 \\
5.530 \\
6.356 \\
6.807 \\
7.486 \\
8.983 \\
10.730 \\
12.909 \\
14.981 \\
15.394\end{array}$ & $\begin{array}{l}210.787,5 \\
265.477,5 \\
235.025 \\
238.350 \\
221.227,5 \\
205.865 \\
202.117,5 \\
187.775 \\
161.362,5 \\
112.357,5 \\
38.485\end{array}$ & $\begin{array}{r}12 \\
14 \\
38 \\
81 \\
106 \\
121 \\
162 \\
188 \\
193 \\
176 \\
148\end{array}$ & $\begin{array}{l}630 \\
665 \\
1.615 \\
3.037,5 \\
3.445 \\
3.327 \\
3.645 \\
3.290 \\
2.412,5 \\
1.320 \\
370\end{array}$ & $\begin{array}{r}5 \\
14 \\
40 \\
70 \\
135 \\
224 \\
353 \\
495 \\
697 \\
925 \\
970\end{array}$ & $\begin{array}{l}262,5 \\
665 \\
1.700 \\
2.625 \\
4.387,5 \\
6.160 \\
7.942,5 \\
8.662,5 \\
8.712,5 \\
6.937,5 \\
2.425\end{array}$ \\
\hline Total & & 98.780 & 2.078 .830 & 1.239 & $23.757,5$ & 3.928 & 50.480 \\
\hline $\begin{array}{c}\% \text { em relação ac } \\
\text { total de óbitos }\end{array}$ & & - & - & 1,2 & 1,1 & 4,0 & 2,4 \\
\hline
\end{tabular}


declarações de óbito não havia referência ao município de residência, em 7 o município referido não constava da lista de cidades do Estado e em 39 o município referido pertencia a outros Estados da Federação. Assim, na análise que se segue, serão considerados apenas os 1.454 óbitos de pacientes com residência determinada no Estado de São Paulo.

A Tabela 4 mostra o local de ocorrência dos óbitos, considerando se a pessoa faleceu no mesmo município em que residiu ou em outro município: $79,2 \%$ dos eventos do Estado ocorreram no município de residência e $20,8 \%$ num município diferente. Dentre as regioes com um mínimo de 50 óbitos, destacam-se São José do Rio Preto com a maior taxa de evasão $(29,1 \%)$ e a Grande São Paulo com a menor taxa $(17,8 \%)$.

Tabela 3. Obitos e coeficiente de mortalidade por doença de Chagas (x 100.000 habitantes), segundo região de residência. Estado de São Paulo, 1987.

\begin{tabular}{lrrr}
\hline \multicolumn{1}{c}{ Residència } & $N^{2}$ & $\%$ & $\begin{array}{c}\text { Coeficiente } \\
\text { de mortalidade }\end{array}$ \\
\hline São Paulo & 551 & 36,3 & 3,4 \\
São Vicente & 14 & 0,9 & 1,0 \\
Taubaté & 15 & 1,0 & 1,0 \\
Sorocaba & 69 & 4,6 & 3,7 \\
Campinas & 135 & 9,0 & 3,2 \\
Ribeirão Preto & 327 & 21,6 & 16,7 \\
São José do Rio Preto & 158 & 10,4 & 15,0 \\
Araçatuba & 46 & 3,0 & 8,6 \\
Presidente Prudente & 29 & 1,9 & 4,4 \\
Marilia & 110 & 7,2 & 7,1 \\
Indeterminado & 7 & 0,5 & $\ldots$ \\
Outros Estados & 39 & 2,6 & $\ldots$ \\
Não referido & 16 & 1,0 & $\ldots$ \\
\hline Total & 1.516 & 100,0 & 4,9 \\
\hline
\end{tabular}

Tabela 4. Concordância entre município de residência $\theta$ ocorrência dos óbitos por doença de Chagas, segundo regiōes. Estado de São Paulo, 1987

\begin{tabular}{|c|c|c|c|c|c|}
\hline \multirow{3}{*}{ Residência } & \multicolumn{5}{|c|}{ Local do óbito } \\
\hline & \multicolumn{2}{|c|}{$\begin{array}{l}\text { Municipio de } \\
\text { residência }\end{array}$} & \multicolumn{2}{|c|}{$\begin{array}{l}\text { Município de } \\
\text { não residência }\end{array}$} & \multirow[t]{2}{*}{ Total } \\
\hline & $\mathrm{N}^{2}$ & $\%$ & No & $\%$ & \\
\hline $\begin{array}{l}\text { São Paulo } \\
\text { São Vicente } \\
\text { Taubaté } \\
\text { Sorocaba } \\
\text { Campinas } \\
\text { Ribeirão Preto } \\
\text { São José do Rio Preto } \\
\text { Araçatuba } \\
\text { Presidente Prudente } \\
\text { Marília }\end{array}$ & $\begin{array}{r}453 \\
9 \\
10 \\
52 \\
107 \\
262 \\
112 \\
33 \\
25 \\
88\end{array}$ & $\begin{array}{l}82,2 \\
64,3 \\
66,7 \\
75,4 \\
79,4 \\
80,1 \\
70,9 \\
71,7 \\
86,2 \\
80,0\end{array}$ & $\begin{array}{r}98 \\
5 \\
5 \\
17 \\
28 \\
65 \\
46 \\
13 \\
4 \\
22\end{array}$ & $\begin{array}{l}17,8 \\
35,7 \\
33,3 \\
24,6 \\
20,6 \\
19,9 \\
29,1 \\
28,3 \\
13,8 \\
20,0\end{array}$ & $\begin{array}{r}551 \\
14 \\
15 \\
69 \\
135 \\
327 \\
158 \\
46 \\
29 \\
110\end{array}$ \\
\hline Total & 1.151 & 79,2 & 303 & 20,8 & 1.454 \\
\hline
\end{tabular}

Nas Tabelas 5 a 7 , foram examinados esses dois conjuntos (classificados pelo local de ocorrência), efetuando a distribuição dos mesmos segundo as variáveis sexo, idade e forma clínica da doença de Chagas. A maior diferença aparece no

Tabela 5. Distribuição dos individuos residentes no Estado que faleceram com doença de Chagas, segundo sexo e local do óbito. Estado de São Paulo, 1987

\begin{tabular}{lcccccc}
\hline & \multicolumn{5}{c}{ Local do óbito } \\
& Sexo & \multicolumn{2}{c}{$\begin{array}{c}\text { Municipio de } \\
\text { residência }\end{array}$} & \multicolumn{2}{c}{ Município de residência } \\
& Ne & $\%$ & $N^{2}$ & $\%$ & \\
& 723 & 62,8 & 213 & 70,3 & 936 \\
\hline Masculino & 428 & 37,2 & 90 & 29,7 & 518 \\
Feminino & 1.151 & 100,0 & 303 & 100,0 & 1.454 \\
\hline Total & & & &
\end{tabular}

Tabela 6. Distribuição dos individuos residentes no Estado que faleceram com doença de Chagas, segundo faixa etária e local do óbito. Estado de São Paulo, 1987

\begin{tabular}{crrrrr}
\hline & \multicolumn{5}{c}{ Local do óbito } \\
Faixa Etária & \multicolumn{2}{c}{$\begin{array}{c}\text { Municipio de } \\
\text { residência }\end{array}$} & \multicolumn{2}{c}{$\begin{array}{c}\text { Município de } \\
\text { não residência }\end{array}$} & Total \\
& $N^{2}$ & $\%$ & $N^{2}$ & $\%$ & \\
\hline $15-19$ & 7 & 0,6 & 3 & 1,0 & 10 \\
$20-24$ & 10 & 0,9 & 4 & 1,3 & 14 \\
$25-29$ & 29 & 2,4 & 6 & 1,9 & 35 \\
$30-34$ & 57 & 5,0 & 18 & 5,8 & 75 \\
$35-39$ & 78 & 6,8 & 20 & 6,5 & 98 \\
$40-44$ & 82 & 7,1 & 32 & 10,4 & 114 \\
$45-49$ & 120 & 11,2 & 38 & 12,3 & 158 \\
$50-54$ & 142 & 12,3 & 38 & 12,0 & 180 \\
$55-59$ & 158 & 13,7 & 28 & 9,1 & 186 \\
$60-64$ & 133 & 11,5 & 41 & 13,4 & 174 \\
$65-69$ & 114 & 9,9 & 32 & 10,4 & 146 \\
70 e + & 207 & 17,9 & 43 & 14,3 & 250 \\
Ignorado & 14 & 0,7 & - & - & 14 \\
\hline Total & 1.151 & 100,0 & 303 & 100,0 & 1.454 \\
\hline
\end{tabular}

Tabela 7. Distribuição dos individuos residentes no Estado que faleceram com doença de Chagas, segundo forma clínica e local do óbito. Estado de São Paulo, 1987

\begin{tabular}{|c|c|c|c|c|c|c|}
\hline \multirow[t]{2}{*}{$\begin{array}{l}\text { Forma } \\
\text { Clinica }\end{array}$} & \multicolumn{2}{|c|}{$\begin{array}{l}\text { Local do } \\
\text { Município de } \\
\text { residência }\end{array}$} & \multicolumn{3}{|c|}{$\begin{array}{l}\text { óbito } \\
\text { Municipio de } \\
\text { não residência }\end{array}$} & Total \\
\hline & $N^{2}$ & $\%$ & $N^{2}$ & $\%$ & $N^{9}$ & $\%$ \\
\hline $\begin{array}{l}\text { Chagas com } \\
\text { comprometimen- } \\
\text { to cardiaco (086.0) } \\
\text { Chagas sem } \\
\text { comprometimen- } \\
\text { to cardiaco }(086.1) \\
(086.2)(086.9)\end{array}$ & $\begin{array}{r}1.051 \\
100\end{array}$ & 91,3 & 272 & 89,8 & 1.323 & 91,0 \\
\hline Total & 1.151 & 100,0 & 303 & 100,0 & 1.454 & 100,0 \\
\hline
\end{tabular}


exame da variável sexo, que revelou um maior percentual de homens no conjunto constituído por pessoas falecidas em município diferente daquele em que residiam, quando comparado ao outro conjunto $(70,3 \% \times 62,8 \%)$; as difcrenças em relação à idade (Tabela 6) e forma clínica (Tabela 7) foram mais discretas.

\section{Discussão}

Comparando-se o número de óbito e a mortalidade proporcional por doença de Chagas em 1987 com a ocorrida em anos anteriores ${ }^{9}$, pode-se afirmar que a tripanossomiase americana mantém a sua tendência de participação no conjunto do obituário do Estado de São Paulo. No presente trabalho, buscou-se contribuir para a caracterização desta magnitude, calculando, além da mortalidade proporcional, o indicador "Anos Potenciais de Vida Perdidos" (APVP). Constatou-se que considerando tão somente a população de 15 a 70 anos - uma vez que não foram registrados óbitos abaixo de 15 anos por doença de Chagas - o indicador APVP mostra uma relativa maior importância da doença de Chagas quando comparada à doença isquêmica do coração (DIC). Essa relativa maior participação da doença de Chagas, quando se utiliza o indicador APVP, é perfeitamente previsível, uma vez que a tripanossomíase americana ocorre em faixas etárias mais jovens; nesse caso, foi valorizada a quantificação dessa diferença, que é de cerca de três vezes na mortalidade proporcional e de duas vezes com APVP. Ambas as nosografias comprometem o aparelho circulatório, apresentando alguns pontos importantes de semelhança $\mathrm{cm}$ relação aos recursos, materiais e humanos, que são dispendidos na assistência médica aos doentes.

Já em relação a distribuição espacial, a publicação do Ministério da Saúde ${ }^{9}$ informa apenas os dados para o Estado de São Paulo como um todo e para o município da Capital. Este estudo limitado da đistribuição da doença não deve ser considerado uma restrição à referida publicação, pois se trata de uma análise efetuada em nível nacional, que tcm exatamente a função de forneccr uma visão abrangente da mortalidade no país. No entanto, considerou-se importante aprofundar essa análise, pois é bastante conhecido que a doença de Chagas teve uma dinâmica de transmissão dif́crenciada no Estado, o que permite supor que a mortalidade seja diferenciada nas diferentes regiões de São Paulo. Como reforço a essa idéia, cabe lembrar, mais uma vez, o clássico estudo de Puffer e Griffith $^{10}$, que destacou a existência de taxas mais elevadas no Município de Ribcirão Preto, em relação ao Município de São Paulo.
Nesse contexto, ao se efetuar a distribuição dos presentes dados, segundo regiōes do Estado (Tabela 3), obtiveram-se dois tipos de informaçōes: o coeficiente de mortalidade específico para cada região e o número absoluto de óbitos. Analisandose em termos de coeficientes, constatou-se que as regiões diferem em relação ao risco de sua população falecer por doença de Chagas. As explicações para essas diferenças devem ser buscadas, num elenco de fatores, dentre os quais destacamse diferenças no nivel de transmissão por vetores no passado, taxas de imigração de infectados diferenciadas e reconhecimento desigual da doença pelos profissionais de saúde das regiões. Com as informações que são obtidas a partir da declaração de óbito, entende-se que é possível apenas registrar esse diferencial nas áreas, e com isso apontar para a necessidade de desenvolvimento de pesquisas que, com dados adicionais, venham explicar a existência desses diferenciais nos coeficientes de mortalidade dessas regiōes.

Analisando-se o número absoluto de óbitos considerado aqui como um segundo tipo de informação, e mais diretamente ligado ao fornecimento de subsídios ao plancjamento da assistência ao chagásico - observa-se que a ordem das regiōes, em termos de frequîcncia, se altera. Fato previsível, uma vez que as populaçðes das regiões não são homogêneas. $O$ que se pretendeu destacar é que este segundo tipo de informação contribui para o conhecimento do dimensionamento da quantidade de recursos, materiais e humanos, que foram - em termos de necessidade - dispendidos para atender a essas pessoas que faleceram com doença de Chagas. Esta nos parece uma dimensão importante em termos do planejamento do atendimento ao chagásico, que evidentemente não deve ser pensado apenas em relação ao subgrupo que faleceu. Em sintese, independentemente de se conhecer qual é o risco de se falecer por doença de Chagas - que é uma informação relevante - deve-se enfatizar que conhecer a dimensão do evento em números absolutos contribui para um melhor equacionamento dos recursos necessários ao atendimento ao chagásico.

É nesta segunda perspectiva, de contribuir para o planejamento do atendimento ao chagásico, que se inserem os dados da Tabcla 4. Nesta Tabela foram calculados, para cada região do Estado, a proporção de pessoas que falcceram em um município diferente daquele no qual residiam. Observou-se que essas proporções variaram de $13,8 \%$ a $35,7 \%$, notando-se, no entanto, uma variação mais estreita $(17,8 \%$ a $29,1 \%)$ nas regiões onde o número de casos foi mais elevado.

Com o propósito de efetuar uma primeira aproximação explicativa dessa mobilidade dos pa- 
cientes chagásicos, examinou-se a composição do grupo de pessoas falecidas em município diferente daquele em que residiu, considerando nesta composição as variáveis sexo, idade e causa do óbito; a seguir, efetuou-se uma comparação das distribuições dessas variáveis com aquelas constatadas nos chagásicos que faleceram nos municípios onde residiam (Tabelas 5,6 e 7). Neste cotejo, a única diferença importante a destacar é a maior proporção de pessoas do sexo masculino no conjunto dos que faleceram em município diverso daquele em que residiam, não havendo, portanto, diferenças relevantes quanto a idade ou formas da doença.

Vir a falecer em outro município pode significar - em termos de assistência médica - duas situações antagônicas: por um lado, um procedimento correto, na medida em que o doente buscou o acesso a recursos médicos necessários c compatíveis com a gravidade do seu caso, incxistentes em seu município; por outro lado, ainda na perspectiva da atenção médica, pode significar um procedimento inadequado na medida $\mathrm{cm}$ que csses recursos, humanos e materiais, cxistiam no próprio municipio de sua residência. Deve-se agregar a essa interpretação, razões de naturcza não ligadas à assistência médica, que podem determinar a decisão da mobilidade da pessoa $\mathrm{cm}$ quesıảo.

Como se vê, trata-se de um problema bastante complexo, que as informaçōes constantes na dcclaração de óbito permitem dimensionar em termos de magnitude, configurando assim um subsídio de interesse ao planejamento da assistência ao chagásico; essas informaçōes permitcm também examinar a distribuição do evento $\mathrm{cm}$ relação ao sexo, idade e causa, como efetuado no presente trabalho. No entanto, parcce evidente que as informações da declaração de óbito não permitem diferenciar - com maior precisão - as diversas situaçőes que determinam a decisão do paciente em buscar assistência médica no município que não reside. Nesse sentido, entende-se mais uma vez que as informações constantes nas Tabclas $4 \mathrm{e}$ 7 permitem basicamente identificar um quadro de distribuição espacial, c ao mesmo tempo demarcar a necessidade de realização de pesquisas, com dados adicionais, que busquem explicar esses comportamentos do grupo populacional chagásico.

Como consideração mais geral, pode-se afirmar que a análise das declaraçōes de obitos onde a doença de Chagas foi citada como causa básica, em 1987, confirmou a importância dessc quadro no Estado de São Paulo, e ao mesmo tempo forneceu informações relativas à distribuição da doença em várias regiões do Estado, quer cm termos de se conhecer o risco da mortalidade da população adulta, quer em termos de plancjamento de atenção ao chagásico. Em relação a cste último ponto, o conhecimento da magnitude constitui uma etapa do plancjamento, devendo ser necessariamente articulada com outras práticas, como por excmplo, a alocação de recursos matcriais e treinamento de pessoal no manejo dos pacientes chagásicos, açōes que devem ser consideradas para todos os nívcis da atenção médica.

LITVOC, J. et al. [Mortality due to Chagas disease in the State of S. Paulo, Brazil: parameter for the establishment of adequate health care for the chagasic patient]. Rev. Saúde públ., S. Paulo, 26: 59-65, 1992. In order to estimate the size of the population with Chagas' discase, an essential parameter for the establishment of an adequate health care policy for this group of patients, data from 1,646 death certificates from the "Fundação Sistema Estadual de Análises de Dados" in which Chagas' disease was mentioned in the State of S. Paulo in 1987 were examined. The population under study comprised adults between 15 and 70 years of age. Objectives also included the determination of Proportional Mortality, Potentially Lost Years of Life and regional distribution of the events in the counties of the State of S. Paulo. Chagas disease accounted for $0.9 \%$ of the totality of the causes of death, Proportional Mortality was $1.2 \%$ and the proportional participation of Chagas' disease in the Potentially Lost Years of Life was $1.1 \%$. Corresponding data for Ischemic Heart Disease were, $4.0 \%$ and $1.2 \%$. $20.8 \%$ of the deaths occurred in counties other than the county of residence, the indexes of evasion varying from $17.8 \%$ to $29.1 \%$ for different regions. Males (22.8\%) more often than females (17.4\%) died outside their county of residence. The largest number of deaths occurred in the metropolitan area of S. Paulo, although Ribeirão Preto county presented the highest mortality indexes.

Keywords: Trypanosomiasis, South American. Mortality. Residence characteristics. Medical care.

\section{Referências Bibliográficas}

1. CAMILO, M. V. F. et al. Análise da triagem de pacientes chagásicos do Hospital das Clínicas - UNICAMP. Rev. Soc. bras. Med. trop., 20 (Supl. 2): 28, 1987.

2. CLASSITTCAÇÃO Estatística Intemacional de Doenças, Lesões e Causas de Óbitos: $9^{a}$ Rev., 1975. São Paulo, Centro da OMS para a Classificação de Doenças cm Português, 1978.

3. DIAS, J. C. P. Doença de Chagas e a questão tecnológica. Bol. Ofic. sanit. panamer. 99: 244-55, 1985.

4. DIAS, J. C. P. Perspectivas de controle da doença de Chagas no IBrasil. Cad. Saúde públ., Rio de Janeiro, 2(1): 84-103, 1986.

5. GOLDBAUM, M. Saúde e trabalho: a doença de Chagas no setor industrial. São Paulo, 1981. [Tesc de Doutorado - Faculdade de Medicina da USP'].

6. GOLDBAUM, M. Doença de Chagas e trabalho em área urbana. São Paulo, 1986. [Disscrtação de Mestrado Faculdade de Medicina da USP]. 
7. GUTMARÃES, C. et al. Mortaljdade de adulto de 15 a 74 anos de idade em São Paulo, Botucatu e São Manuel (Brasil), 1974/1975. Rev. Saúde públ., S.Paulo, 13 (Supl. 2), dez. 1979.

8. LAURENTI, R. et al. Estatísticas de saúde. São Paulo, EPU/Ed. USP, 1985.

9. MINISTÉRIO DA SAÚDE. Secretaria Nacional de Açōes Básicas de Saúde. Divisão Nacional de Epidemiologia. Estatísticas de mortalidade: Brasil, 1977 a 1985. Brasília, Centro de Documentação do Ministério da Saúde, 1980-1988.

10. PUFFER, R. R. \& GRIFFITH, G. W. Características de la mortalidad urbana. Washington, DC, Organizacion Panamericana de la Salud, 1968. (OPAS - Publicacion Cientifica, 151)
11. ROMEDER, J. M. \& McWHINNIE, J. R. Potential years of life lost between ages 1 and 70: and indicator of premature mortality for health planning. Int. J. Epidem., 6(2): 143-51, 1977.

12. WALDMAN, E. A. et al. Freqüência de portadores de infeç̧ão chagásica e de antígeno HBS, em doadores de sangue de alguns municípios do Estado de São Paulo. In: Congresso Sociedade Brasileira Medicina Tropical, 18² Ribeirão Preto, 1982. Anais. Ribeirão Preto, 1982. A-17 [Resumo]

Recebido para publicaçāo em 17/4/91 Reapresentado 14/11/91 Aprovado para publicaçấo em 19/11/91 